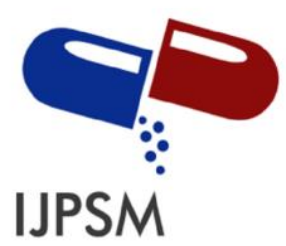

Priyanka V. Bagade et al, Int. Journal of Pharmaceutical Sciences and Medicine (IJPSM),

Vol.6 Issue. 6, June- 2021, pg. 28-33

ISSN: 2519-9889

Impact Factor: 3.426

\title{
Formulation and Evaluation of Gel Based Herbal Hand Wash Using Extracts of Argemone Mexicana
}

\author{
Priyanka V. Bagade ${ }^{1}$; Nikita D. Gidde ${ }^{2}$; Sohel I. Nadaf ${ }^{3}$; Priyanka V. Desai ${ }^{4}$; \\ Mayuri S. Lokhande ${ }^{5}$; Lingraj V. Sargar ${ }^{6}$ \\ 1,2,3,4,5 Rajarambapu College of Pharmacy, Kasegaon (MS) India 415404 \\ ${ }^{6}$ Bharati Vidyapeeth college of Pharmacy, Kolhapur (MS) India \\ Email id - Bagadepriyanka88@gmil.com \\ DOI: 10.47760/ijpsm.2021.v06i06.003
}

\begin{abstract}
The primary purpose of developing an herbal hand wash with a gel basis is to promote "personal hygiene." Hand hygiene is a requirement and one of the most critical processes in the preparation of meals, food service, and house and other day care facilities. On the market, antibacterial hand wash is composed of alcohol-based cleaning products, which have a number of disadvantages. An effort was made to develop a gel-based herbal hand wash utilising Argemone Mexicana extracts to avoid the adverse effects of synthetic hand wash preparations such as itching, dryness, irritation, and dermatitis. The antibacterial activity of the prepared gel hand wash toward E. coli, S. aureus, and P. aeruginosa was determined using the Agar plate diffusion technique. Physical and chemical characteristics such as pH, Viscosity, Foam height, Foam retention, Anti-Microbial Activity, Skin irritation assessment, and other parameters were used to evaluate herbal hand wash, and the results were found to be within normal ranges with minimum to no adverse effects.
\end{abstract}

Keywords: Herbal hand wash, Herbal extracts, Argemone Mexicana, Hygiene, antimicrobial activity

\section{Introduction}

Hands are the major route of microbe and illness transfer; hand cleanliness is the most efficient way to prevent the spread of hazardous germs and diseases. In healthcare, hand cleanliness is the best and most effective, simplest, and affordable technique to prevent nosocomial infections ${ }^{[1]}$. Contaminated hands can function as vectors for the spread of germs. Outbreaks are conveyed from one human to another when a food handler contaminates his or her hands and then transfers these bacteria to customers via hand contact with food or drinks. ${ }^{[2]}$. The user is exposed after ingesting these germs, which might cause gastrointestinal disease. Microorganisms infiltrate the food supply when people handle ready-to-eat foods. ${ }^{[3]}$.

The hands of healthcare providers are the main cause of the spread of multidrug-resistant bacteria and sickness to patients. As an outcome, it presents the issue of hygienic hand cleansing ${ }^{[4]}$. Various antimicrobial compounds are now accessible as alcohol-based hand wash, detergent, and other items on the market. These soaps or solutions aid in the prevention of health-care-associated microbiological contamination, although they come with certain disadvantages or adverse reactions. Their usage on a regular basis might promote skin irritation and infection resistance. ${ }^{[4,5]}$. 


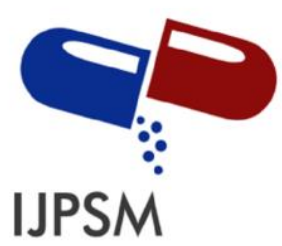

Priyanka V. Bagade et al, Int. Journal of Pharmaceutical Sciences and Medicine (IJPSM), Vol.6 Issue. 6, June- 2021, pg. 28-33

ISSN: 2519-9889

Impact Factor: 3.426

Plant species are the earliest resource of pharmacologically active molecules, and they have provided humans with a wide range of medicinal purposes beneficial substances for ages ${ }^{[6]}$. Plants have a large number of antiinfective chemicals. Plant-based antimicrobials represent a large unexplored source of medication. They cure bacterial infections effectively while preventing several of the adverse reactions with synthetic antimicrobial agents. Flavonoids and polypeptides found in modern medicinal herbs have been found to be efficient against a wide spectrum of bacteria. ${ }^{[4,7]}$.

In the present research Argemone Mexicana has been selected as the major component in a gel-based herbal antibacterial hand cleanser. Argemone Mexicana Linn (Papaveraceae) is a widespread plant which grows along sides of the roads and in fields across India. Argemone Mexicana Linn, a member of the Papaveraceae family and often referred as "Mexican prickly poppy" and "Satyanashi," is a common weed in agricultural and wastelands. ${ }^{[8]}$.

\section{Material and Methods}

\section{Material}

Among the most crucial substances employed in the current investigation to develop a gel-based herbal hand wash was Argemone Mexicana. Argemone Mexicana leaf was harvested in the Sangli district of Maharashtra. The specimens were authenticated by YCP, Karad College. Triethanolamine, cabopol-934, Sodium lauryl sulphate, Methyl paraben, Rose Water, and perfume were procured from RCP in Kasegaon, Maharashtra. Chemicals of analytical grade are employed in the preparation.

\section{Bacterial strains}

Escherichia coli (gram-ve) ATCC 10531

Pseudomonas aeruginosa (gram-ve) ATCC 25619

Staphylococcus aureus (gram +ve) ATCC 6538

\section{Method}

\section{Preparation of Extracts ${ }^{11}$}

In a $100 \mathrm{ml}$ methanol solution, $10 \mathrm{~g}$ of dry plant material was added ( 9 parts of methanol: 1 part of water). This combination was warmed for 12 to 24 hours in a water bath at $60^{\circ} \mathrm{C}$, filtered, and plant extract was extracted.

\section{Formulation of Gel Based Herbal Hand Wash using extracts of Argemone Mexicana Preparation of gel base:}

Herbal gel was prepared overnight using deionized water and 1 percent w/w carbopol-934 as a gelling agent. To achieve homogeneous polymer dispersion, the swollen polymer was then agitated using a mechanical stirrer. By adding modest volumes of triethanolamine and stirring continuously, the $\mathrm{pH}$ was raised to $7.0^{[6]}$.

\begin{tabular}{|c|c|c|c|}
\hline Sr. no. & Ingredients & Quantity taken & Role \\
\hline $\mathbf{1}$ & Cabopol-934 & $0.25 \mathrm{gm}$ & Jelling agent \\
\hline $\mathbf{2}$ & Purified Water & $20 \mathrm{ml}$ & Vehicle \\
\hline $\mathbf{3}$ & Triethanolamine & q.s & Neutralizer \\
\hline
\end{tabular}

Table No.1: Preparation of Herbal Gel Base for Herbal Hand Wash. 


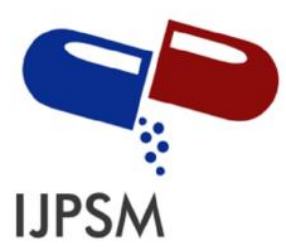

Priyanka V. Bagade et al, Int. Journal of Pharmaceutical Sciences and Medicine (IJPSM),

Vol.6 Issue. 6, June- 2021, pg. 28-33

ISSN: 2519-9889

Impact Factor: 3.426

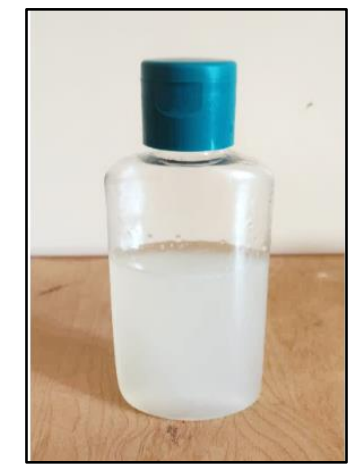

Fig 1: Gel base for herbal hand wash

\section{Formulation of gel based herbal hand wash}

As reported in Tables 1 and 2, the extract of Argemone mexicana was subsequently added to this base to develop a gel-based hand wash preparation. The formulation was put to an organoleptic test.

The following formula was used to develop the gel based herbal hand wash.

\begin{tabular}{|c|c|c|}
\hline Sr. no. & Ingredients & Quantity taken \\
\hline $\mathbf{1}$ & Gel base & $30 \mathrm{ml}$ \\
\hline $\mathbf{2}$ & Extract of Argemone mexicana & $1 \mathrm{gm}$ \\
\hline $\mathbf{3}$ & Sodium lauryl sulphate & $0.3 \mathrm{gm}$ \\
\hline $\mathbf{4}$ & Methyl paraben & $0.1 \mathrm{gm}$ \\
\hline $\mathbf{5}$ & Rose Water & $10 \mathrm{ml}$ \\
\hline
\end{tabular}

Table No. 2: Preparation of Gel Based Herbal Hand wash

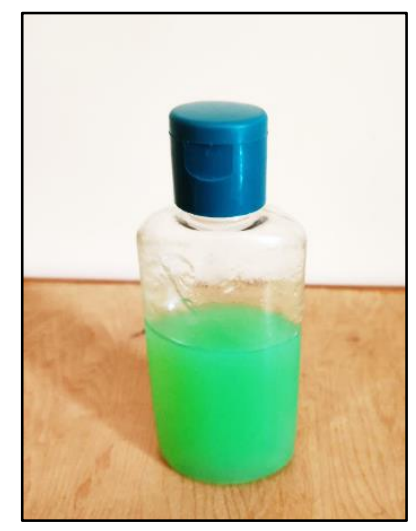

Fig 2 : Gel based herbal hand wash 


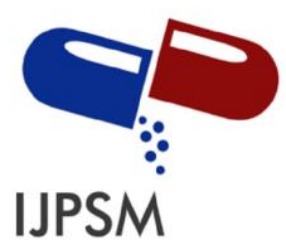

Priyanka V. Bagade et al, Int. Journal of Pharmaceutical Sciences and Medicine (IJPSM), Vol.6 Issue. 6, June- 2021, pg. 28-33

ISSN: 2519-9889

Impact Factor: 3.426

\section{Evaluation of Gel Base for Herbal Hand Wash}

\section{Physical Evaluation}

Visual inspection of the Poly-Herbal Gel Based Hand Wash was performed. The test parameters were colour, odour, texture., Appearance and Homogeneity

\section{pH}

In 100 millilitres of distilled water, $1 \mathrm{gm}$ of gel-based herbal hand wash was mixed. The $\mathrm{pH}$ of the mixture was examined using a previously standardised digital $\mathrm{pH}$ metre.

\section{Viscosity}

The thickness of a gel-based herbal hand cleanser was tested using a digital Brookfield viscometer.

\section{Irritancy Test:}

During irritancy checks, there is no redness, edema, inflammation, or discomfort in the preparation. These things are absolutely safe to use on the skin.

\section{Foam Height}

In $50 \mathrm{~mL}$ of distilled water, $1 \mathrm{gm}$ of gel-based herbal hand wash was dispersed. Fill a $500 \mathrm{ml}$ poured measuring cylinder halfway with water to make it $100 \mathrm{ml}$. The foam height was measured above the aqueous volume after 25 strokes were administered and let to stand until the aqueous volume reached $100 \mathrm{ml}$.

\section{Foam Retention}

$50 \mathrm{ml}$ of the gel-based herbal hand wash was agitated 10 times in a $250 \mathrm{ml}$ graduated cylinder. The quantity of foam was monitored at 1-minute periods for 4 minutes. Foam retention should be stable over a period 5 minutes.

\section{Spreadability ${ }^{[9]}$}

The spreadability device was made out of a wooden block with a pulley attached to one end. To measure spreadability, this method evaluated the slip and drag properties of gels.

An excess of gel (nearly $2 \mathrm{~g}$ ) from the research was applied to this ground slide. After that, the gel was placed between this slide and a glass slide with the same proportions as the fixed ground slide, as well as a hook.

A $1 \mathrm{~kg}$ weighted plate was placed on top of the two slides for 5 minutes to release air and create a homogeneous gel coating between them. Excess gel was pulled away from the edges. The top plate was then pulled with an 80-gram pull. With the help of the string linked to the hook, the time (in seconds) taken for the top slide to traverse a distance of $7.5 \mathrm{~cm}$ may be estimated. When the time is shorter, spreadability is easier.

Spreadability was calculated using the following formula:

$$
S=M \times \frac{L}{T}
$$

Where, $\mathrm{S}=$ Spreadability, $\mathrm{M}=$ Weight in the pan (tied to the upper slide), $\mathrm{L}=$ Length moved by the glass slide and $\mathrm{T}=$ Time (in sec.) taken to separate the slide completely each other. 


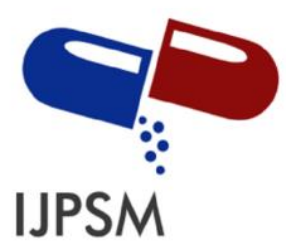

Priyanka V. Bagade et al, Int. Journal of Pharmaceutical Sciences and Medicine (IJPSM),

Vol.6 Issue. 6, June- 2021, pg. 28-33

ISSN: 2519-9889

Impact Factor: 3.426

\section{In-Vitro Antimicrobial Activity by Agar Plate Diffusion Method:}

Using the agar well diffusion procedure, antimicrobial screening was done on the produced herbal gel hand wash. The microorganisms examined were E. coli, S. aureus, and P. aeruginosa. Sterile filter paper discs with a diameter of $6 \mathrm{~mm}$ were filled with 10 litres of herbal hand wash. The sterile discs were designed to completely absorb the hand wash. The discs were placed on agar that had previously been infected with the test culture when they were completely dry. To determine the inhibitory zone, the plates were incubated at $37^{\circ} \mathrm{C}$ for 24 hours.

\section{Results and Discussion}

All the observation data for physical and chemical evaluations of herbal hand wash presented as follows :

\begin{tabular}{|c|c|c|}
\hline Sr.no & Parameters tested & Result \\
\hline & Color & Greenish \\
\hline & Odor & Characteristic \\
\hline & Texture & Smooth \\
\hline & Appearance & Translucent \\
\hline & Homogenicity & good \\
\hline & pH of solution & 6.61 \\
\hline & Viscosity (cps) & 50 c Pascal's \\
\hline & Irritancy & no redness, edema, infection, or no \\
& Foam height & $435 \mathrm{ml}$ \\
\hline & Foam retention at 5 min & $21.5 \mathrm{ml}$ \\
\hline & Spredability & good \\
\hline
\end{tabular}

Table No. 3: Evaluation Parameters of Gel Based Herbal Hand wash

\section{In-Vitro Antimicrobial Activity by Agar Plate Diffusion Method ${ }^{[10]}$}

In this evaluation test, the antimicrobial activity of herbal hand wash was found to be good, and the gel-based herbal hand wash created possesses antimicrobial activity towards species of bacteria like E. coli, S. aureus, and P. aeruginosa. The preparation had a good zone of inhibition extending from 16 to $23 \mathrm{~mm}$, as shown in the table below:

\begin{tabular}{|c|c|}
\hline Organism & Zone of inhibition $(\mathbf{m m})$ \\
\hline E. Coli & 16 \\
\hline S. aureus & 23 \\
\hline P. aeruginosa & 19 \\
\hline
\end{tabular}

Table No 4: Antimicrobial Screening of prepared formulation 


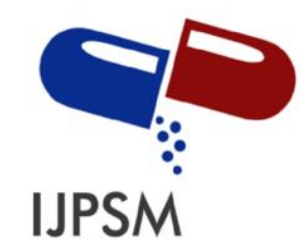

Priyanka V. Bagade et al, Int. Journal of Pharmaceutical Sciences and Medicine (IJPSM), Vol.6 Issue. 6, June- 2021, pg. 28-33

ISSN: 2519-9889

Impact Factor: 3.426

\section{Conclusion}

Natural remedies are regarded to be more suitable than synthetic therapies since they are safer and have lesser adverse effects. Herbal preparations are gaining popularity on the international market. Argemone Mexicana extract is being used to make a herbal gel-based hand cleanser. As per the observations of many researches, herbs are highly beneficial agents that may be used as hand wash with reduced adverse effects and longerlasting benefits. The data indicate that the gel composition is homogenous. These herbal hand wash compositions outperformed widely available alcohol-based hand wash preparations. Pathogenic bacteria such as Staph aureus, E. coli, and Pseudomonas aeruginosa have been demonstrated to be effective towards Staph aureus, E. coli, and Pseudomonas aeruginosa, with no negative effects on human tissue.

\section{References}

[1]. Ravi K, Pratibha MD, Kolhapure SA. 2005;Evaluation of the antimicrobial efficacy and safety of Pure Hands as a hand sanitizer: Indian Journal of Clinical Practice, 15(10),19-27.

[2]. Mashood Ahmed Shah, Satheesh Babu Natarajan, Mohd. Gousuddi , 2014; Formulation, Evaluation and Antibacterial Efficiency of Herbal Hand Wash Gel. Int. J. Pharm. Sci. Rev. Res., 25(2),120-124.

[3]. National Disease Surveillance Centre. Preventing Food borne Disease: A Focus on the Infected Food Handler. 2004.17-20.

[4]. Ali Heyam Saad, Shehab Naglaa Gamil, Rasool Bazigha and Rana Samour1, 2011; Formulation and evaluation of herbal hand wash from Matric aria chamomilla flowers extracts. IJRAP,2 (6) ,1811-1813.

[5]. Pittet D. 2005, Clean hands reduce the burden of disease. Lancet; 366: 185-187.

[6]. Powar P. V, Bhandari N.R, Arya Ashwini, Sharma P. H., 2015; Formulation and Evaluation of Poly Herbal Anti-Bacterial Gel Based Hand Wash. Int. J. Pharm. Sci. Rev. Res., 33(1),79-82.

[7]. Abbiw DK. Useful plants of Ghana - West African use of wild and cultivated plants. Intermediate Technology Publications and the Royal Botanic Gardens Kew; 1990.

[8]. Saurabh Rajvaidhya, B.P.Nagori, G.K.Singh ,B. K. Dubey, Prashant Desai and Sanjay Jaina, 2012; review on argemone Mexicana linn - an Indian medicinal plant. International journal of pharmaceutical sciences and research. (01), 2494-2501.

[9]. Kumar L, Verma R, 2010, In-vitro evaluation of topical gel prepared using natural polymer. Int. Drug Delivery. 2, 58-63.

[10].Minakshi G. Joshi, D V Kamat and S D Kamat, 2008; Evaluation of herbal formulation, Natural product radiance 7(5);413-415.

[11].Nikita d. Gidde *, priyanka v. Desai, priyanka v. Bagade, seema u. Shinde, manojkumar m. Nitalikar., 2021, formulation and evaluation of herbal hand sanitizer using argemone mexicana and calendula officinalis plant extract, international research journal of pharmacy, 12 (4), 34-36. 